Tohoku J. exp. Med., 1983, 141, Suppl., 427-430

\title{
Epidemiological Studies on Renal Papillary Necrosis with Diabetes Mellitus in Japan
}

\author{
Yasuo Oshima and Syo Isogai \\ The Second Department of Internal Medicine, Toho \\ University School of Medicine, Tokyo 143, Japan
}

\begin{abstract}
Oshima, Y. and IsogaI, S. Epidemiological Studies on Renal Papillary Necrosis with Diabetes Mellitus in Japan. Tohoku J. exp. Med., 1983, 141, Suppl., 427-430 — Eighty-six cases of renal papillary necrosis (RPN) published in Japan over the years 1949-1980 were studied. While several hundreds cases of RPN were reported in certain European countries and the USA, only 5 cases were publisished in Japan up until 1960. However, the number of case reports has increased to 28 over the 5 year period of 1976-1980. The ratio of diabetics (62 cases) to nondiabetics ( 24 cases) is about $2.6: 1$ and is the same as that reported by Lauer in the USA. In diabetics, RPN was more frequently seen in females (3.4: 1) and at more advanced age. Frequency of bilateral occurrence was high (47.5 $\%$ ). The prevalence of the association of glomerulosclerosis $(75.0 \%)$ was higher than that reported by Silverman et al. in the USA $(47.1 \%)$. The Incidence of the association of disturbances of consciousness $(29.5 \%)$ was similar to that reported by Edmondson et al. It was concluded that the number of cases of RPN with diabetes mellitus in Japan started to increase about 20 years later than in the USA, - and that the frequency of the association between RPN and diabetic glomeruloscleosis in Japan seems to be higher than in the USA. Furthermore, it was noted that RPN was frequently associated with hyperosmolar nonketotic diabetic coma. _ renal papillary necrosis ; diabetic glomerulosclerosis ; hyperosmolar nonketotic diabetic coma
\end{abstract}

Although prior to 1960 , many reports on renal papillary necrosis (RPN) were published in European countries and the USA ${ }^{1-3)}$, only a few cases of RPN were reported in Japan. However, after 1961, the number of case reports increased from year to year, especially in association with diabetes. The present study was designed to elucidate the epidemiological and clinical features of RPN in Japan.

\section{Method}

The subjects were 86 cases of RPN reported in Japan over the years 1949 to 1980 . Their diagnosis had been made by autopsy in 30 cases, nephrectomy in 8 , fragments of necrotic medullary tissue in urine in 7, pyelography in 33 and unknown in 8 cases. Their ages ranged from 16 to 77 years. Diabetes was present in 62 cases. The results obtained in this epidemiological study on the Japanese were compared with those of several reports from the USA, and statistical analysis was performed uisng the $\chi^{2}$-test. 


\section{Results}

Incidence and sex differences in $R P N$. The number of reported cases were $2,3,11$, 20, 22 and 28 cases between 1949-1955, 1956-1960, 1961-1965, 1966-1970, 19711975 and 1976-1980 respectively. The ratio of males to females was $1: 3.5$.

Frequency of the association of $R P N$ with diabetes. Sixty-two out of 86 cases were associated with diabetes. The ratio of diabetics to nondiabetics was $2.6: 1$. Incidence and sex differences in RPN with diabetes. The number of cases of RPN with diabetes were $3,9,15,16$ and 19 between 1956-1960, 1961-1965, 1966-1970, 1971-1975 and 1976-1980 respectively. Females were affected more than males through the period. The ratio of males to females was $1: 3.4$ (Fig. 1).

Frequency of the association of RPN with diabetic glomerulosclerosis. Diabetic glomerulosclerosis was described in 30 of 40 cases $(75.0 \%)$ studied (Fig. 2).

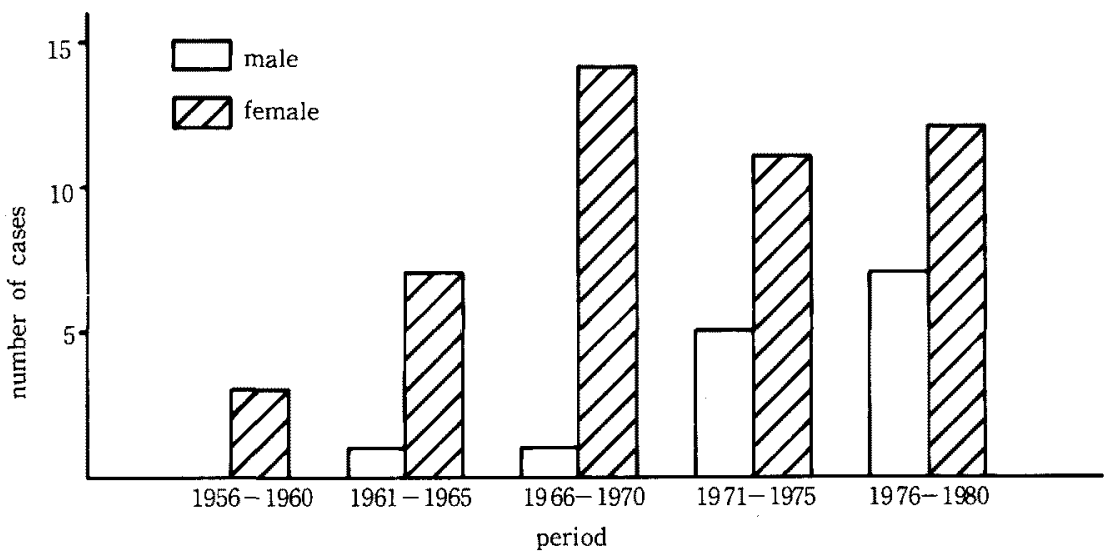

Fig. 1. Incidence and sex difference in renal papillary necrosis with diabetes mellitus in Japan.

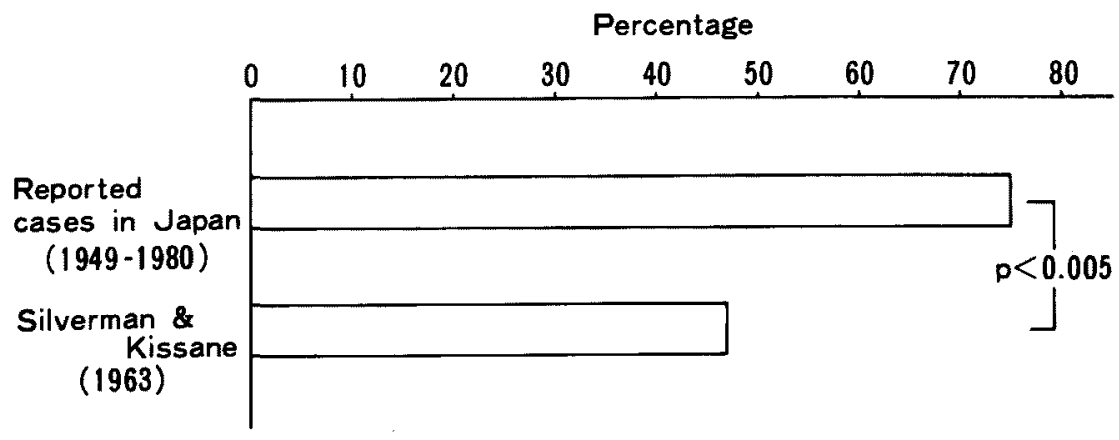

Fig. 2. Frequency of the association of renal papillary necrosis with diabetic glomerulosclerosis.

Japan : $75.0 \%(30 / 40)$. USA : $47.1 \%(48 / 102)$. 


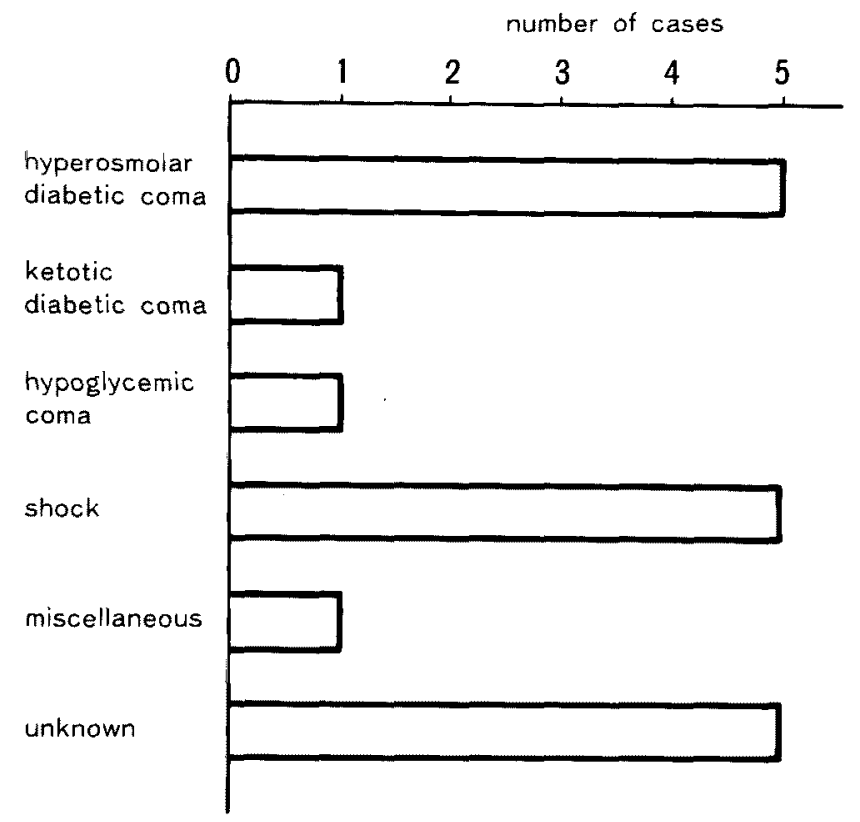

Fig. 3. The number of cases of disturbance of consciousness associated with renal papillary necrosis in diabetics. Eighteen out of 62 cases had disturbances of consciousness due to various causes.

Incidence of disturbances of consciousness in RPN with diabetes. Incidence of disturbances of consciousness associated with RPN in diabetics was $29.0 \%$ (18 out of 62 cases). Hyperosmolar nonketotic diabetic coma (HNKDC) was observed in 5 , ketotic diabetic coma (KDC) in 1, hypoglycemic coma in 1, septic shock in 5, miscellaneous in 1 and unknown in 5 cases (Fig. 3).

Comparison of these results with those of other countries. The incidence of RPN in females was higher than that in the USA, and the frequency of bilateral RPN was slightly lower than that reported by Lauler et al. (1960). The frequency of association of RPN with diabetic glommrulosclerosis was higher than reported by Silverman and Kissane ${ }^{3)}$ in the USA. No significant difference was seen between the frequency of the association of RPN with disturbances of consciousness in diabetics in our study and in that reported by Edmondson et al. ${ }^{1}$.

\section{Discussion}

The number of cases of RPN with diabetes started to increase about 20 years later in Japan than in European countries and the USA. This increase might be due to the increase in the number of diabetics of prolonged duration and in glomerulosclerosis. RPN is well known to be highly associated with diabetes and diabetic glomerulosclerosis. Our data were similar to data from the USA for frequency of association of RPN with diabetes. However, we found that the incidence of RPN with diabetic glomerulosclerosis was more frequent than in the 
USA. The reason for this is unknown, but it is suspected that Japanese patients, especially females with diabetic glomerulosclerosis, are liable to infection of the urinary tract.

It has been reported that RPN tended to be associated with disturbances of consciousness, especially with diabetic coma and septic shock. Our present study showed a high incidence of association between RPN and disturbances of consciousness; however, our data were similar to data published by Edmondson et al. ${ }^{1)}$ in the USA. Also, it was noted that there were more cases of HNKDC than of KDC. The reason for this is unknown, but it might be that dehydration, which reduces the renal blood flow, was more severe in HNKDC than in KDC. Moreover, dehydration might be induced by both HNKDC and RPN ${ }^{4)}$. Therefore, we cannot conclude at present whether HNKDC is a cause or a result of RPN.

\section{References}

1) Edmondson, H.A., Martin, H.E. \& Evance, N. (1974) Necrosis of renal papillae and acute pyelonephritis in diabetes mellitus. Arch. intern. Med., 79, 148-175.

2) Lauler, D.P., Schreiner, G.E. \& David, A. (1960) Renal medullary necrosis. Amer. J. Med., 29, 132-156.

3) Silverman, J.L. \& Kissane, J.M. (1963) Diabetic glomerulosclerosis in renal papillary necrosis. An autopsy study. Amer. J. clin. Path., 40, 532-536.

4) Oshima, Y., Inoguchi, T., Sakai, Y., Takane, T., Isogai, S., Kawamura, T., Tamura, K. \& Kawamura, S. (1980) A case of hyperosmolar, nonketotic diabetic coma probably due to renal papillary necrosis. $J$. med. Soc. Toho, 27, 472-476. 